\title{
Coupling MCNP-DSP and LAHET Monte Carlo Codes for Designing Subcriticality Monitors for Accelerator-Driven Systems
}

T. Valentine ${ }^{1}$, Y. Rugama ${ }^{2}$, J. Munõz-Cobos ${ }^{3}$, and R. Perez ${ }^{4}$

1 Oak Ridge National Laboratory P. O. Box 2008 Oak Ridge, TN 37831 USA

Phone: 865-574-6176, Fax: 865-574-6182, newline email: valentinete@ornl.gov

2 Poly. Tech. Univ. of Valencia, Chemical and Nuclear Engineering Department APDO. 22012, Valencia, Spain 46071

Phone: 34-96-387-76-30, Fax: 34-96-387-76-39, email: yrugama@iqn.upv.es

3 Poly. Tech. Univ. of Valencia, Chemical and Nuclear Engineering Department APDO. 22012, Valencia, Spain 46071

Phone: 34-96-387-76-30, Fax: 34-96-387-76-39, email: jlcobos@iqn.upv.es

4 Oak Ridge National Laboratory P. O. Box 2008 Oak Ridge, TN 37831 USA

Phone: 865-574-5571, Fax: 865-574-1249, email: zxi@ornl.gov

\begin{abstract}
The design of reactivity monitoring systems for accelerator-driven systems must be investigated to ensure that such systems remain subcritical during operation. The Monte Carlo codes LAHET and MCNP-DSP were combined together to facilitate the design of reactivity monitoring systems. The coupling of LAHET and MCNP-DSP provides a tool that can be used to simulate a variety of subcritical measurements such as the pulsed neutron, Rossi- $\alpha$, or noise analysis measurements.
\end{abstract}

\section{Introduction}

Accelerator-driven spallation neutron sources are an attractive and safe option for waste transmutation of material from commercial power reactors. The cost effectiveness of accelerator-driven fissile systems is highly dependent on the degree of subcriticality of the system core and hence dependent on the ability to control and maintain the system core at a specific subcritical state. Conventional control of commercial nuclear reactors is made possible by the presence of negative reactivity feedback mechanisms and delayed neutrons, which slow down the reactor dynamic response to easily manageable levels and the fact that the neutron flux or power is directly related to the reactivity of the system. For waste transmutation and fissile material disposition, however, its is often desirable to harden the neutron spectrum, which could make control a difficult problem because the inherent natural feedback mechanisms that are common in thermal reactors are reduced. Control of accelerator-driven fissile targets is made possible by operating the system in a subcritical state and controlling the reactor power by adjusting the beam current. For these and other reasons, significant research efforts are ongoing in both Europe and the U. S. to address issues related to accelerator-based actinide waste burners. 
Concerns about the degree of subcriticality of an accelerator-driven fissile system have mandated that methodologies be developed to measure the subcriticality of the system. The Polytechnic University of Valencia and Oak Ridge National Laboratory undertook an effort to develop a computational tool to investigate the time-dependent response of neutron detectors in an accelerator-driven system. This is the first step necessary to develop a measurement methodology. This paper provides a brief description of the research performed to develop a time-dependent Monte Carlo calculation for accelerator-driven systems.

\section{Monte Carlo Methods}

The computation of the time-dependent detector responses in an acceleratordriven system is best accomplished using Monte Carlo programs. Monte Carlo codes preserve the exact nature of particle interactions within a fissile system and are only limited by the ability to describe the geometry of the fissile system and by the nuclear data to describe the particle interaction probabilities.

The accelerator-driven system of interest injects protons into a target assembly to produce high-energy neutrons. These high-energy neutrons slow down in the target assembly and transmute the nuclear waste. The Monte Carlo code LAHET [1] is used to simulate the interactions of the protons within the target region to produce high-energy neutrons. The LAHET code also simulates the slowing-down of the high-energy neutrons. The Monte Carlo program MCNPDSP [2] is required to simulate the interactions for neutrons with energies less than $20 \mathrm{MeV}$. This code is used to estimate the counting statistics for neutron and/or gamma ray counters in fissile systems. Therefore, the coupling of LAHET and MCNP-DSP provides a means to estimate the time-dependent response of neutron and/or gamma ray detectors that may be used in the design of a subcriticality monitoring system for an accelerator-driven system.

\section{Monte Carlo Code MCNP-DSP}

The Monte Carlo code MCNP-DSP was developed from the MCNP [3] Monte Carlo code to simulate a variety of subcritical measurements. In MCNP-DSP, the variance reduction features were disabled to obtain a strictly analog particle tracking to follow the fluctuating processes more accurately. Because typical biasing techniques are employed to reduce the variance of estimates of first moment quantities, they do not preserve the higher moments; therefore, analog Monte Carlo calculations must be performed when analyzing subcritical measurements whose measured quantities are directly related to the higher moments of the neutron populations. Because the use of average quantities reduces the statistical fluctuation of the neutron population, average quantities such as the average number of neutrons from fission are not used; instead, appropriate probability distribution functions are sampled. The prompt particle tracking begins with the source event. The source particles and their progeny are tracked to their 
exticntion by either absorption or escape. During the particles' histories, appropriate interactions within the detectors produce detection events, and the time of the detection events are tracked to produce time-dependent detector responses. These time sequences are sampled into blocks of 512 or 1024 data points. The blocks of data are then processed according to the type of measurement being simulated. Each data block can be Fourier transformed and a complex multiplication performed to obtain estimates of auto and cross spectra from the detector responses. The auto and cross spectra are then averaged with previous estimates until the desired number of data blocks have been accumulated.

\section{LAHET}

The Monte Carlo code LAHET was developed by Los Alamos as a general tool for simulating the transport of nucleons, pions, and muons. In the simulation of accelertor-driven systems, the LAHET code is used to track the interaction of protons within a target and to track the subsquent neutrons produced from the spallation events. Neutrons with energies greater than $20 \mathrm{MeV}$ are tracked using LAHET. The data for neutrons with energies less than $20 \mathrm{MeV}$ are written to a file to be tracked using MCNP-DSP. The neutron's position, direction, energy, and time are provided in the output file produced by LAHET. The data in LAHET are broken down into batches. A given number of protons are produced in each batch with varying numbers of neutrons produced for each batch.

\section{Use of LAHET Data in MCNP-DSP}

MCNP-DSP treats each batch from the LAHET file as a pulse from the proton injector. The number of source particles per pulse (per batch) is specified in the LAHET calculation. Likewise, the initial time distribution of the proton pulse is specified in LAHET. There are additional inputs required in the MCNPDSP input file and in the extra MCNP-DSP input file. The user must supply the frequency of the proton injector. The simulation a ssumes that the injector behaves as a pulsed source with a fixed period between the pulses. The time of the pulse depends on the number of proton pulses that occur in each data block. The data block is the time over which the detector responses are accumulated and is equal to the number of detector time bins divided by the sampling rate as specified in the MCNP-DSP extra input file. If there is only one proton pulse per block the starting time of the injector signal is sampled uniformly in the data block. This simulates the use of an internal clock on a processor to start the data accumulation. If more than one proton pulse occurs per block, then the starting time $\left(t_{s}\right)$ of the pulse is sampled uniformly between 0 and $T_{p p}$ where $T_{p p}$ is the period between pulses and is equal to the inverse of the proton pulse frequency. The time of the subsequent pulses is determined by $t_{s}+i \cdot T_{p p}$ where $i$ is the $i^{\text {th }}$ pulse after the first. An option is included in MCNP-DSP to allow the proton injector to serve as a trigger resulting in only one pulse per block occurring at time zero. 
The number of data blocks that are acquired are determined by the number of pulses per block and the total number of batches in the LAHET input file. The energy, position, direction, weight, time, and particle type are provided in the LAHET output file. This information is used to determine the location of the particle in the MCNP-DSP model. If the particle cannot be found anywhere in the geometry the particle will be skipped. If the source particle time is greater than the period of the data block, then the particle is also skipped. These are the only factors that will result in the omission of a particle from being tracked.

A simulation of an accelerator-driven system [4] was performed using the LAHET source file with MCNP-DSP. This simulation was performed to determine the cross spectra between the proton injector and a detector in the system. The cross spectra can then be used to determine the prompt neutron decay constant for the system. The prompt neutron decay constant is directly related to the subcriticality of the system. An example of the cross spectra between the proton injector and the system detector for a single pulse per data block is provided in Figure 1.

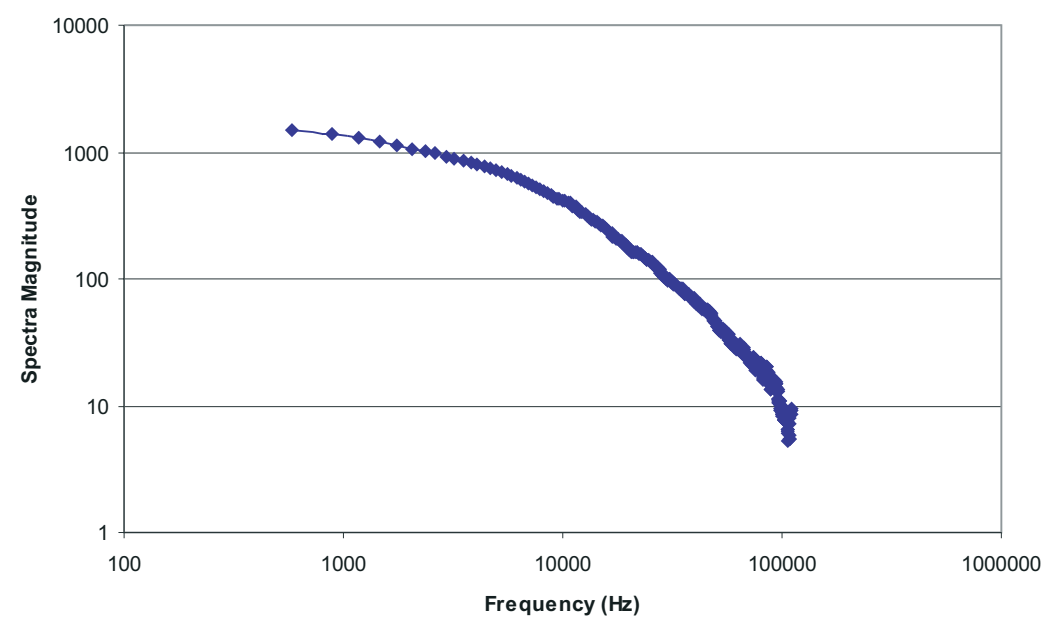

Fig. 1. Source-detector cross spectra for accelerator-driven system.

\section{Summary}

The coupling of LAHET with MCNP-DSP provides a general tool for the calculation of the time dependent detector response for an accelerator-driven system. The coupling is achieved by reading a binary LAHET file that describes the particle's energy, position, direction, weight, time, and type of particle. MCNP-DSP uses this information to determine the location of the particle in the MCNP-DSP model. MCNP-DSP models the accelerator-driven system with the proton injec- 
tor signal as one of the measured quantities. MCNP-DSP allows for two possible computation modes depending on the use of the proton injector signal.

Acknowledgement This work was performed at Oak Ridge National Laboratory which is managed by UT-Battelle, LLC, under contract DE-AC05-00OR22725 with the U.S. Department of Energy.

Disclaimer-The submitted manuscript has been authored by a contractor of the U. S. Government. Accordingly, the U. S. Government retains a nonexclusive, royalty-free license to publish or reproduce the published form of this contribution, or allow others to do so, for U. S. Government purposes.

\section{References}

1. Richard E. Prael and Henry Lichtenstein," User Guide to LCS: The LAHET Code System," LA-UR-89-3014, Los Alamos National Laboratory, 1989.

2. Valentine, T.E., "MCNP-DSP Users Manual," ORNL/TM-13334, Oak Ridge Nat. Lab, January 1997.

3. Briesmeister, J. F., Ed., "MCNP4A-A General Monte Carlo N-Particle Transport Code," LA-12625-M, Los Alamos National Laboratory, 1993.

4. Rugama, Y., Muñoz-Cobo, J. L., and Valentine, T. E., "Noise Method for Monitoring the Subcriticality in Accelerator Driven Systems," Proceedings at the Monte Carlo 2000 Conference, Lisbon, Portugal, Oct. 23-26, 2000. 